\title{
Awareness and utilization of cervical cancer screening services among primary health care providers in Zaria
}

\author{
Anisah Yahya, ${ }^{1}$ Zubair Hadi Ismail, ${ }^{2}$ Umma Suleiman Bawa ${ }^{1}$ \\ ${ }^{1}$ Department of Obstetrics and Gynaecology and ${ }^{2}$ Department of Radiotherapy and Oncology, College of \\ Medical Sciences, Ahmadu Bello University, Zaria, Nigeria
}

\begin{abstract}
Cervical cancer screening services have led to a significant decrease in the incidence of cervical cancer in countries were such services are implemented as population-based services. Cervical cancer still remains the most commonly diagnosed cancer in several countries in Sub-Saharan Africa and South Eastern Asia. This study explored the awareness, utilization and provision of cervical screening services among Primary Health Care providers in Zaria, Nigeria. A qualitative study was conducted among Primary Health Care providers in Zaria Local Government Area Kaduna State Nigeria. Five Primary Health Care centres were purposively selected for the study. Data was collected using Focus Group discussion method. The data from the discussion sessions were recorded on
\end{abstract}

Correspondence: Anisah Yahya, Department of Obstetrics and Gynaecology, College of Medical Sciences, Ahmadu Bello University, Zaria/Ahmadu Bello University Teaching Hospital, P.M. B 06 ShikaZaria, Kaduna state. Nigeria.

Tel.: +2348035925175.

E-mail: anisahy@yahoo.com

Key words: Cervical cancer screening, Primary Health Care providers.

Contributions: AY, design, definition of intellectual content, literature search, experimental studies, manuscript preparation, concept, manuscript editing, manuscript review; ZHI, definition of intellectual content, manuscript editing, manuscript review; USB, manuscript editing, manuscript review.

Conflict of interest: The authors declare no potential conflict of interest.

Funding: None.

Conference presentation: Paper presented at the 59th Conference of the West African College of Surgeons in Dakar, Senegal 21-24 January 2019.

Received for publication: 11 February 2019.

Revision received: 26 December 2019

Accepted for publication: 26 December 2019.

This work is licensed under a Creative Commons Attribution NonCommercial 4.0 License (CC BY-NC 4.0).

(C) Copyright: the Author(s), 2019

Licensee PAGEPress, Italy

Annals of African Medical Research 2019; 2:68

doi:10.4081/aamr.2019.68 tape and transcribed verbatim to capture the exact messages. The transcripts were then analysed into themes. Majority of the participants were within 30-39-year age range, 75\% were females, 64.3\% were Community Health Extension Workers' and $42.9 \%$ had $>10$ years working experience. The major themes were awareness of cervical cancer, awareness of cervical cancer screening methods, knowledge about cervical cancer screening methods and utilization. Many participants were not well informed about cervical cancer screening methods. Uptake among female participants was also low. In light of these findings, there is a need to train the health personnel in Primary Health Care centres on cervical cancer screening so that they can adequately educate women on cervical cancer screening.

\section{Introduction}

The International Agency for Research on Cancer reported a worldwide estimate of 569,847 new cases of cervical cancer in 2018 and 311,365 deaths occurring from the disease in $2018 .{ }^{1}$ It is the fourth most common cancer among women worldwide and the fourth most common cause of cancer death among women worldwide in $2018 .^{2}$ Cervical cancer is second only to breast cancer as a cause of cancer incidence in low- and middle-income countries. ${ }^{3}$ It is the commonest cancer among women in several countries of Sub-Saharan African and South Eastern Asia. In Nigeria, it was estimated that 14,943 new cases of cervical cancer occurred in 2018 with 10,407 deaths from cervical cancer. ${ }^{2}$

Premature deaths from cervical cancer have been reported in Central Europe, Eastern Europe and Central Asia. ${ }^{4}$ A similar trend has also been reported in Uganda and Zimbabwe. ${ }^{5,6}$ This has been attributed to lack of effective screening programmes for early detection and treatment of premalignant lesions of the cervix. Implementation of population based cervical cancer screening programmes and treatment of pre-invasive lesions of the cervix have led to a significant decline in the incidence rates of cervical cancer in the developed countries. ${ }^{7}$

In Nigeria, cervical cancer screening is largely opportunistic. ${ }^{8}$ Screening is mainly limited to tertiary centres where women that present for consultation due to other medical conditions are screened for cervical cancer. Some centres also screen women routinely after the postnatal period. Non-governmental organizations and some professional bodies also organize periodic screening programmes for their members and sometimes the rural communities. Such approaches to screening are ineffective in cervical cancer prevention and control as they lack the potential to reach out to the whole population.

Primary Health Care (PHC) centres provide health care services at the grassroots in most communities. Women within the reproductive age group utilize such centres for various reproduc- 
tive health needs like contraceptive services and antenatal care services. Primary Health Care providers can offer health education on cervical cancer screening to women that present to them for various reproductive health needs. The aim of this study was to assess the awareness, utilization and provision of cervical screening services among Primary Health Care providers in Zaria, Nigeria.

\section{Materials and Methods}

\section{Study area}

The study was conducted in Zaria Local Government Area of Kaduna state in North Western Nigeria. There are urban, semi urban and rural settings within the Local Government Area. There are 11 Primary Health Care centres within Zaria with a total of 155 technical staff. The staff includes 125 Community Health Extension Workers, 18 Nurses and 12 Community Health Officers (Zakari 2018: Personal communication). Other staff includes laboratory technicians, undergraduates and informally trained ad hoc staff. There are also secondary, tertiary and many private health care facilities within Zaria Local Government Area. The Primary Health Care centres in Zaria Local Government Area lack facilities for cervical cancer screening. However, there are services for cervical cancer screening and treatment of premalignant lesions of the cervix in the tertiary health centre in Zaria Local Government Area.

\section{Study design and sampling method}

This was a qualitative study. Five Primary Health Care centres in Zaria Local Government Area of Kaduna state were purposively selected after due consultation with the department of Primary Health Care of Zaria Local Government. These centres cut across urban, semi-urban and rural settlements. All health personnel present during the focus group discussions in the chosen centers participated in the focus group discussion irrespective of sex and cadre. This is because all staff are involved in provision of health care services. The focus group discussions were conducted in a quiet room at the various centers. The discussions were done on round tables. The tape recorders were kept on the table to enable clear recording. A total of 28 health care personnel from the 5 centers that were chosen for the study participated in the discussions. Each group discussion had between 5 to 6 health care providers as participants. The research team included the principal researchers, lead moderator and note taker

\section{Data collection}

Five Focus group discussions were conducted to obtain data. The staff in charge of each Primary Health Care center that participated made arrangements for the Focus Group Discussion sessions. The discussions were conducted from 12/04/2018 to 19/04/2018. Three groups had 6 participants while the other 2 groups had 5 participants each. The research team conducted the Focus Group Discussions and comprise of the principal researchers, lead moderator and note taker. The research team comprise of both males and females with experience and training in research. The research team had no prior relationships with study participants. The note taker recorded the time the discussions began and the time they ended. The discussion lasted between 45 to 60 minutes.

\section{Data management}

The discussions were tape recorded and transcribed verbatim.
The transcribed data was analysed into themes. The themes are awareness of cervical cancer and cervical cancer screening, Knowledge of the available methods of cervical cancer screening, Uptake of cervical cancer screening among participants, Skills for offering any of the methods for cervical cancer screening and Willingness to offer cervical cancer screening services to women.

\section{Ethical consideration}

Ethical clearance to conduct the research was given by the department of Primary Health Care of Zaria Local Government Area. Consent was also obtained from all participants before commencement of the Focus Group Discussions.

\section{Results}

\section{Awareness of cervical cancer}

All the participants appear to be aware of cervical cancer. Even though the extent of their awareness varied. Some participants have had family members who had suffered cervical cancer while some had provided care to clients previously diagnosed with cervical cancer. Social media dominated participants' views as sources of information about cervical cancer.

\section{Awareness of cervical cancer screening methods.}

Most of the participants said that it is possible to detect cervical cancer before symptoms develop. The main reason given by most of the participants for cervical cancer screening was early detection of the disease. None of the participants mentioned detection of pre-cancerous lesions and treatment or detecting high risk human Papilloma Virus (HPV) as the reason for screening for cervical cancer. Some of the responses given by the participants were:

"Yes, it can be detected before a woman develops symptoms", (FGD 1, participant 13, 38 years).

"The importance of screening is to detect the disease at an early stage" (FGD 1, participant 2, 28 years).

"The main aim and importance of these methods is to confirm the disease" (FGD 4, participant 49, 29 years).

Most of the participants mentioned Pap smear and HPV testing as the cervical cancer screening method they were aware of. Others mentioned ultrasound scan, magnetic resonance imaging (MRI), $\mathrm{X}$-rays, high vaginal swab and biopsy as methods of cervical cancer screening.

"Screening methods include investigations in the hospital such as X-ray and ultrasound scan" (FGD 3, participant 4, 50 years)

\section{Knowledge of the available methods of cervical cancer screening}

This theme explored the participants' knowledge about the cervical cancer screening methods that they were aware of. Pap smear was the commonest screening method for cervical cancer known to the participants. When asked what type of test a Pap smear is, some said it was a histopathological test that can detect cancer cells. Most of the participants said they knew that samples are taken from the cervix but they do not know what is done with it and how it is interpreted. Their source of information about cervical cancer screening was mainly during their training as health workers.

Some participants believe that only women within the reproductive age should have a Pap smear done while few felt that only women with symptoms of cervical cancer should have a Pap smear done. 
"Any woman of child bearing age should have a Pap smear done" (FGD 4, participants 1, 35 years).

"Any woman that has symptoms and signs of cervical cancer" (FGD 1, participant 1, 44 years).

Participants were also asked about how frequent Pap smears should be done. Many said they were not sure while some mentioned 1 year and 3 years as the interval for Pap smear screening.

When participants were asked whether they do Pap smears for their clients, they mentioned that none of them had ever done a Pap smear for any woman. The reason given was mainly lack of skills for Pap smear screening in their centers.

"I have never done a Pap smear for my patient, but I refer patients to general hospital or teaching hospital for proper diagnosis" (FGD 2, participant 1, 44 years).

"I don't know how to do Pap smear and have not referred any patient for the test" (FGD 3, participant 5, 38 years).

With regards to awareness on HPV testing, most of the participants said they have heard about the test however they did not know how it is being performed and interpreted. Some said that the test can detect the cells that cause cervical cancer. The main source of information about HPV test was media.

Participants were then asked about Visual inspection with acetic acid (VIA) and Visual inspection with Lugol's iodine (VILI) i.e. the 'see and treat approach'. Majority said they have never heard about it. Few said they have heard about it but could not remember much.

"I have heard about it but cannot remember much about it" (FGD 2, participant 6, 20 years).

\section{Utilization of cervical cancer screening among partici- pants}

Only one out of the 21 female participants has ever had Pap smear for cervical cancer screening. However, most of them have planned to have a Pap smear whenever they have the opportunity.

"I did a Pap smear test during visit by some medical people for screening at the community" (FGD 3, participant 5, 25 years).

"I have not done it but I will do it whenever I get the opportunity" (FGD 3, participant 6, 32years)

The main reasons for not screening by the female participants were mainly due to lack of screening services in their health centers and busy work schedule. Some of the participants mentioned that they are afraid of having a positive test.

"There are no facilities for cervical center screening unless we go to the teaching hospital which is very far and usually there is a very long queue" (FGD 2, participant 5, 33 years).

\section{Skills for offering any of the methods of cervical cancer screening}

All the participants mentioned that they lack the necessary skills for performing any of the cervical cancer screening methods. They mentioned that they have never had any hands on training on cervical cancer screening methods either during training as health workers or in form of workshops during their years of service.

"We have never had training on cervical cancer screening" (FGD 5, participant 4, 40 years).

"We heard about cervical cancer during workshop on family planning, but we have never had training on cervical cancer screening" (FGD 1, participant 4, 30 years).

\section{Willingness to offer cervical cancer screening services to women}

All participants said that they are willing and ready to be trained on cervical cancer screening methods.

"We are willing to have the training to get more knowledge about the disease" (FGD 1, participant 6, 33 years).

"Yes, very willing to have training on Pap smear and HPV test to improve our knowledge on patient care" (FGD 5, participant 3, 33 years).

\section{Discussion}

The median age of participants was 38 years. Most of them were females $(75 \%)$ (Table 1$)$, thus, most of the participants can develop cervical cancer. They also fall within the age range for cervical cancer screening which is 21 to 65 years according to the World Health Organisation. ${ }^{9}$ There was no doctor among the participants and only few of the participants were nurses. This shows uneven distribution of health personnel within the primary health care centers that participated in this research. The median duration of service is 10 years $(\mathrm{SD}=5.8)$ (Table 1$)$. This suggests that they have fairly long years of service ahead thus they can be trained to offer cervical cancer screening services.

Awareness of cervical cancer was high among participants. This is not surprising because they are health personnel. Similar finding has also been reported in many parts of the developing nations. ${ }^{10-13}$

Even though most of the participants mentioned Pap smear and HPV tests as screening tests for cervical cancer, some participants had poor knowledge about cervical cancer screening methods. Magnetic resonance imaging, X-ray and ultrasound scan were mentioned as screening methods for cervical cancer screening. Health care providers are an important source of health information and therefore expected to educate the public on important health issues. Misconception and ignorance among health care workers can be a barrier to effective health education and subsequent prevention. There was also lack of awareness with regards to visual inspection with acetic acid (VIA) and visual inspection with Lugol's iodine (VILI) 'the see and treat approach' method of screening for cervical cancer. This is also surprising because the

Table 1. Sociodemographic profile of participants.

\begin{tabular}{lcc} 
& Frequency & Percentage \\
Age & & \\
$21-30$ & 4 & 14.3 \\
$31-40$ & 15 & 53.6 \\
$41-50$ & 8 & 28.6 \\
$>50$ & 1 & 3.6 \\
Total & 28 & 100 \\
Sex & & \\
Female & 21 & 75.0 \\
$\quad$ Male & 7 & 25.0 \\
\hline Qualification & & \\
Nurse & 2 & 7.1 \\
$\quad$ Community health worker & 4 & 14.3 \\
Community health extension worker & 18 & 64.3 \\
$\quad$ Bachelor of science & 1 & 3.6 \\
$\quad$ Laboratory technician & 3 & 10.7 \\
Total & 28 & 100 \\
Years of service & & \\
$\quad<10$ & 16 & 57.1 \\
$\quad$ To & 12 & 42.9 \\
$\quad$ Total & 28 & 100 \\
\hline
\end{tabular}


World Health Organisation (WHO) recommends the use of VIA/VILI for cervical cancer screening in low resource settings. ${ }^{1}$ However, the fact that there are no standard protocols for such service in our primary health centers may account for the ignorance. Low awareness about VIA as a screening tool for cervical cancer has also been reported among health workers in other clinical settings. ${ }^{12,14}$ Singh et al. ${ }^{14}$ reported that knowledge about VIA was low $(7 \%)$ among nurses working in tertiary institutions of rural India. This may be due to the availability of Pap smear in such centers which have higher sensitivity and specificity as a screening tool for cervical cancer. Dulla et al. ${ }^{12}$ also reported low awareness (37.6\%) of VIA among female health workers in Southern Ethiopia. A similar study by Gharoro et al.$^{10}$ on awareness of Pap smear among female health workers in a tertiary institution in Nigeria reported that awareness varies significantly across different categories of health workers. The higher value obtained by Dulla et al. ${ }^{12}$ when compared to the study by Singh et al. ${ }^{14}$ may be due to the fact that the study by Dulla et al. involved all cadre of female health workers across all levels of health care while Singh et al involved only nurses in their study.

Despite the fact that the participants were aware of Pap smear and HPV test as screening methods for cervical cancer, they demonstrated lack of knowledge about how to perform the tests, when and how often the test should be performed. A similar finding by Pegu et al. ${ }^{11}$ showed that awareness of cervical cancer screening was high among nurses in a tertiary hospital, however they were not aware of routine screening guidelines and had limited understanding of the different types of cervical cancer screening techniques. Awodele et al. ${ }^{15}$ also reported good knowledge of cervical cancer but limited understanding of the types of cervical cancer screening techniques among nurses in Lagos, Nigeria.

The participants also lack the skills to offer such services. This is likely due to the fact that none of them has ever had any form of hands on training on cervical cancer screening apart from lectures that they had during their training as health workers. A study on awareness on cervical cancer screening among nurses reported that majority of nurses felt that speculum examination and Pap smear are doctors' procedure. ${ }^{14}$ This belief may limit them from offering such services even where there is indication.

Uptake of screening was very low from this study (4.8\%). The participant that screened for cervical cancer in this research did so during an outreach programme organized by a Non-governmental organization. This reflects the general low uptake of cervical cancer screening in Nigeria ${ }^{16}$ and the opportunistic screening that characterizes cervical cancer screening in Nigeria. ${ }^{8}$ Low uptake of cervical cancer screening has been reported among different population of women ranging from health care personnel in clinical settings ${ }^{10,14-16}$ to women at grass root level in our communities. ${ }^{17-20}$ Reasons given for not having Pap smear were mainly due to the absence of such services in most of their facilities and their tight work schedule. However, the fact that most of them have planned on having a Pap smear done means that making such services readily available will likely improve uptake rates.

Participants were willing to be trained on cervical cancer screening so that they can offer the service to women in the communities. The public health care implication of this is that establishing cervical cancer screening services at Primary Health Care level will likely help in reducing the burden of the disease.

\section{Conclusions}

Awareness about cervical cancer screening among the partici- pants did not necessarily translate into good knowledge about cervical cancer screening methods. There was poor knowledge with regards to the guidelines of the cervical cancer screening methods that were known to the participants. There was apparent insufficient knowledge about VIA and VILI which has been recommended for low resource settings by the WHO. Uptake of cervical cancer screening was also low. All these findings can create a barrier for effective cervical cancer control coupled with the fact that Nigeria lacks a standard protocol for cervical cancer screening. The recent guideline developed by the Society of Obstetrics and Gynaecology of Nigeria can be operationalized at Primary Health Care centers to bridge this gap. Also, there is a need for training of Primary Health Care providers for them to acquire the necessary skills needed to cater for the health care need of their communities. The major limitation of this work is the small sample size and the fact that it was conducted in only one local government area and thus not representative of what obtains in other parts of the country.

\section{References}

1. Bray F, Ferlay J, Soerjomataram I, et al. Global cancer statistics 2018: GLOBOCAN estimates of incidence and mortality worldwide for 36 cancers in 185 countries. CA Cancer J Clin 2018;68:394-424.

2. Arbyn M, Weiderpass E, Bruni L et al. Estimates of incidence and mortality of cervical cancer in 2018: a worldwide analysis. The Lancet. https://doi.org/10.1016/S2214-109X(19)30482-6.

3. Bhatla N, Aoki D, Sharma DN, Sankaranarayanan R. Cancer of the cervix. Int J Gynecol Obs 2018;143:22/36.

4. Bray F, Lortet Tieeulent J, Znacr. Patterns and trends in Human Papilloma Virus related diseases in Central and Eastern Europe and Central Asia. Vaccine 2013;3:H32.

5. Wabinga HR, SN, PM A, et al. Trends in the incidence of cancer in kampala, Uganda. 1991-2010. Int J Cancer 2014;135: 432-9.

6. Chokunong E, Borok MZ, Chirenje ZM, Nyakabau AM PD. Trends in the incidence of cancer in black population of Harare, Zimbabwe. 1991-2010. Int J Cancer 2013;133:721-9.

7. Kim J, Edmunds W, Goldie S. Modelling cervical cancer prevention in the developed countries. Vaccine. 2008;23:59-62.

8. Ndikom CM, Ofi BA. Awareness, perception and factors affecting utilization of cervical cancer screening services among women in Ibadan, Nigeria: a qualitative study. Reprod Health 2012;9:11-8.

9. World Health Organization. Comprehensive Cervical Cancer Control: A Guide to Essential Practice. 2nd ed. WHO Library Cataloguing-inPublication Data. WHO Press, Geneva, Switzerland. 2014. 2014.

10. Gharoro EP, Ikeanyi EN. An appraisal of the level of awareness and utilization of the Pap smear as a cervical cancer screening test among female health workers in a tertiary health institution. Int J Gynecol Cancer 2006;16:1063-8.

11. Pegu B, Dhima N, Chatervedi J, Sharma S. Nurses Knowledge and attitude regarding cervical cancer screening test among female health workers in a tertiary health institution. Int $\mathrm{J}$ Reprod Contraception Obstet Gynaecol 2017;6:907-10.

12. Dulla D, Daka D, NW. Knowledge about Cervical cancer screening and its practice among female health workers in Southern ethiopia. A Cross sectional study. Int J Womens Health 2017;9:365-72.

13. Assoumou SZ, Mabika BM, Mbiguino AN, et al. Awareness 
and knowledge regarding of cervical cancer, Pap smear screening and human papillomavirus infection in Gabonese women. BMC Womens Health 2015;15:1-7.

14. Singh E, Seth R, Rani V, Srivastava D. Awareness of cervical cancer screening among nursing staff in a tertiary institution in India. J Gynecol Oncol 2012;23:141-6.

15. Awodele O, Adeyomoye A, Awodele D, et al. A Study on Cervical Cancer Screening Amongst Nurses in Lagos University Teaching Hospital, Lagos, Nigeria. J Cancer Educ 2011;26:497-504.

16. Oluwole EO, Mohammed AS, Akinyinka MR, Salako O. Cervical Cancer Awareness and Screening Uptake among Rural Women in Lagos, Nigeria. J Commun Med Prim Heal
Care 2017;29:81-8.

17. Yahya A, Mande AT. Awareness and Knowledge of cervical cancer and its screening methods among women attending primary health health care centers in Zaria, North Western Nigeria. Trop J Obstet Gynaecol 2019;36:271-5.

18. Ahmed SA, Sabitu K, Idris SH, Rukaiya A. Knowledge, attitude and Practice of cervical cancer among market women in Zaria, Nigeria. Niger Med J 2013;54:316-9.

19. Eze J, Umeora O, Obuna J, Al E. Cervical cancer awareness and cervical screening uptake at Mater Misericordiae Hospital, Afioko, South East Nigeria. Ann Afr Med 2012;11:238-43.

20. Ezem B. Awareness and uptake of cervical cancer screening in Owerri, South Eastern Nigeria. Ann Afr Med 2007;6:94-8. 